\title{
The nuclear poly(A) polymerase and Exosome cofactor Trf5 is recruited cotranscriptionally to nucleolar surveillance
}

\author{
MAXIME WERY, ${ }^{1}$ SABINE RUIDANT, ${ }^{1}$ STÉPHANIE SCHILLEWAERT, NATHALIE LEPORÉ, \\ and DENIS L.J. LAFONTAINE \\ Fonds de la Recherche Scientifique (FRS-FNRS), Académie Wallonie-Bruxelles, Institut de Biologie et de Médecine Moléculaires, Université Libre \\ de Bruxelles, Charleroi-Gosselies, B-6041 Belgium
}

\begin{abstract}
Terminal balls detected at the $5^{\prime}$-end of nascent ribosomal transcripts act as pre-rRNA processing complexes and are detected in all eukaryotes examined, resulting in illustrious Christmas tree images. Terminal balls (also known as SSU-processomes) compaction reflects the various stages of cotranscriptional ribosome assembly. Here, we have followed SSU-processome compaction in vivo by use of a chromatin immunoprecipitation (Ch-IP) approach and shown, in agreement with electron microscopy analysis of Christmas trees, that it progressively condenses to come in close proximity to the $5^{\prime}$-end of the $25 \mathrm{~S}$ rRNA gene. The SSU-processome is comprised of independent autonomous building blocks that are loaded onto nascent pre-rRNAs and assemble into catalytically active pre-rRNA processing complexes in a stepwise and highly hierarchical process. Failure to assemble SSU-processome subcomplexes with proper kinetics triggers a nucleolar surveillance pathway that targets misassembled pre-rRNAs otherwise destined to mature into small subunit $18 \mathrm{~S}$ rRNA for polyadenylation, preferentially by TRAMP5, and degradation by the $3^{\prime}$ to $5^{\prime}$ exoribonucleolytic activity of the Exosome. Trf5 colocalized with nascent pre-rRNPs, indicating that this nucleolar surveillance initiates cotranscriptionally.
\end{abstract}

Keywords: ribosome synthesis; pre-rRNA processing; Exosome; cotranscriptional assembly; U3 snoRNP/SSU processome; nucleolus

\section{INTRODUCTION}

Ribosome synthesis is a complex multistep process that starts in the nucleolus with the transcription by RNA polymerase I (Pol I) of a polycistronic transcript (35S in yeast) that encodes three out of the four mature rRNAs (18S-5.8S-25S rRNAs). Each rRNA is interspersed with noncoding spacer sequences that require precise excision by processing (Supplemental Fig. S1). The fourth rRNA (5S) is independently transcribed by Pol III. Concurrent with prerRNA synthesis and processing, an exquisite series of modification and assembly reactions take place (for review, see Henras et al. 2008). Ribosome synthesis that aims to faithfully assemble four rRNAs and $\sim 80$ r-proteins involves no less than $\sim 200$ protein and $\sim 200$ snoRNA trans-acting

\footnotetext{
${ }^{1}$ These authors contributed equally to this work.

Reprint requests to: Denis L.J. Lafontaine, Fonds de la Recherche Scientifique (FRS-FNRS), Académie Wallonie-Bruxelles, Institut de Biologie Moléculaire et de Médecine Moléculaires, Université Libre de Bruxelles, Rue Professors Jeener and Brachet, 12, B-6041 CharleroiGosselies, Belgium; e-mail: denis.lafontaine@ulb.ac.be; fax: +3226509747.

Article published online ahead of print. Article and publication date are at http://www.rnajournal.org/cgi/doi/10.1261/rna.1402709.
}

factors. This activity is a major cellular undertaking and entails a great deal of coordination and integration (for review, see Jorgensen et al. 2004). The transient association of scores of ribosome synthesis factors with nascent transcripts, resulting in nascent pre-ribosomes, generates macromolecular structures visualized by electron microscopy (EM) on chromatin spreads as terminal balls.

Initially detected in the late 1970 s by Miller, terminal balls are now known to act as pre-rRNA processing complexes and are observed on rRNA gene transcripts across eukaryotes, in all tissues and organisms examined, providing illustrious Christmas trees textbook images (Mougey et al. 1993, and references therein). A recent characterization of the Christmas trees in yeast indicates that terminal balls are entities whose dynamics reflects the various stages of ribosome cotranscriptional assembly. Terminal balls are first detected as small $5^{\prime}$-terminal knobs $(\sim 15-20 \mathrm{~nm})$, that most likely involve the box $\mathrm{C}+\mathrm{D}$ snoRNA U3, and which then condense into larger knobs ( $\sim 40 \mathrm{~nm})$, comprising the entire 18S rRNA sequence (Osheim et al. 2004). In fast growing yeast cells, the compaction of pre-18S rRNA sequences into a large particle 
is extremely rapid and occurs within seconds of completion of its transcription. Pre-18S rRNA compaction is followed by cotranscriptional cleavage within ITS1 (the spacer sequence that separates the $18 \mathrm{~S}$ and $5.8 \mathrm{~S}$ rRNAs; Supplemental Fig. S1) and pre-40S subunit release. The frequency of cotranscriptional cleavage ( $\sim 50 \%$ in fast growing yeast) varies according to growth rates and is responsive to stress. After cleavage, a new terminal knob is formed on the nascent large subunit rRNA, compacting it progressively in a $5^{\prime}$ to $3^{\prime}$ direction into a pre-60S ribosome (Osheim et al. 2004).

A large U3 snoRNP particle, also referred to as SSUprocessome or $90 \mathrm{~S}$ pre-ribosome, was characterized several years ago that not only contained the canonical box $\mathrm{C}+\mathrm{D}$ snoRNP proteins and several U3 specific proteins but $\sim 40$ additional U-three-associated proteins or Utps, all required for $40 \mathrm{~S}$ ribosome synthesis (Dragon et al. 2002; Grandi et al. 2002). Large terminal knobs did not form upon depletion of U3 or individual Utps (Dragon et al. 2002; Gallagher et al. 2004).

The SSU-processome is comprised of independent autonomous building blocks that are loaded onto nascent pre-rRNAs and assemble into catalytically active pre-rRNA processing complexes in a stepwise and highly hierarchical process. Preassembled ribosome building blocks likely allow minimizing the huge complexity of ribosome assembly. To date, three SSU-processome subcomplexes have been discriminated structurally and/or functionally: UTP-A (also referred to as t-UTP for transcription UTP), UTP-B (or PWP2), and UTP-C (or CURI), each comprised of $\sim 6-$ 7 proteins (Dosil and Bustelo 2004; Gallagher et al. 2004; Krogan et al. 2004; Perez-Fernandez et al. 2007; Rudra et al. 2007). UTP-A consists of Utp4, Utp8, Utp9, the HEAT/ Armadillo-repeats Utp10, Utp15, Utp17/Nan1, and Pol5 (Krogan et al. 2004). t-UTP only differs from UTP-A by the presence of Utp5. UTP-A binds very early to nascent prerRNAs in a step that impinges on further SSU-processome components recruitment, including that of U3 itself (PerezFernandez et al. 2007). It is not yet known whether UTP-B performs a specific function (Champion et al. 2008). UTP$\mathrm{C}$ integrates pre-rRNA processing and r-protein genes transcription (Rudra et al. 2007).

Yeast strains with severe ribosome synthesis defects generally fail to accumulate high levels of pre-rRNAs, indicating that active surveillance mechanisms prevail. These at least include the targeting of misassembled prerRNAs, recognized by as yet unknown mechanisms, for polyadenylation by the nuclear poly(A) polymerase TRAMP complexes and selective $3^{\prime} \rightarrow 5^{\prime}$ exoribonucleolytic digestion by the Exosome (Dez et al. 2006, 2007; for review, see Houseley et al. 2006). The TRAMP complexes are comprised of a zinc knuckle and putative RNA binding protein (either Air1 or Air2), a DExD/H-box family helicase (Mtr4/Dob1), and a poly(A) polymerase (either Trf4 or Trf5), the later defining the TRAMP4 or TRAMP5 complexes, respectively (LaCava et al. 2005; Vanacova et al. 2005; Wyers et al. 2005; for reviews, see Houseley et al. 2006; Andersen et al. 2008). The Exosome consists of 10 core subunits of which Rrp44/Dis3 is directly active in RNA synthesis and degradation, carrying both exoRNase and endoRNase activities (Dziembowski et al. 2007; Schneider et al. 2007; Lebreton et al. 2008). Specific cofactors allow distinguishing the activities of the cytoplasmic versus nuclear forms of the Exosome. A specific nuclear cofactor of the Exosome is the RNase T/D family protein Rrp6 that carries hydrolytic exoRNase activity.

Here, we describe a chromatin immunoprecipitation (Ch-IP)-based approach to analyze in vivo the cotranscriptional steps of pre-ribosome assembly. Further, we report that alteration in the kinetics of SSU-processome subcomplexes assembly activates a nucleolar surveillance pathway that targets pre-rRNA otherwise destined to form the small ribosome subunits for polyadenylation, preferentially by TRAMP5, and degradation by the Exosome. The colocallization of Trf5 at the rDNA transcription unit makes it likely that this surveillance at least initiates cotranscriptionnally.

\section{RESULTS}

\section{Christmas tree visualization in vivo by $\mathrm{Ch}$-IP}

Members of the UTP-A/t-UTP subcomplex of the SSUprocessome had previously been localized by Ch-IP at the rDNA both in yeast and humans (Gallagher et al. 2004; Prieto and McStay 2007). Initial analysis in yeast exclusively focused on the $5^{\prime}$-ETS region of the rDNA, which encodes the binding sites of U3. Initially we were interested to test whether $\mathrm{t}$-Utps interacted with other regions of the rDNA. We also wanted to address whether other subclasses of SSU-processome components interacted with the rDNA.

First, we established the profile of interaction with the rDNA of Rpa190, the largest subunit of Pol I, by a standard Ch-IP procedure followed by qPCR analysis (Fig. 1). As expected, Pol I was distributed evenly throughout the transcription unit, starting at the promoter region (amplicon 6) and ending at the terminator (amplicon 18) (Fig. 1B). The signal was at its strongest level at the $5^{\prime}$-ETS pair and decreased progressively toward the $3^{\prime}$-end of the rDNA gene. Otherwise wild-type untagged isogenic strains showed no significant interaction at any of the amplicons tested. Tagging Rpa190 with HA or TAP provided similar profiles of interaction with the rDNA, and most further experiments used the TAP epitope. All TAP fusions were generated at the carboxyl-terminal end of the target genes by homologous recombination and integration of suitable PCR cassettes in the genomes of haploid yeast cells. Growth of the TAP-tagged strains was indistinguishable from the untagged wild-type, indicating that the fusion proteins were fully functional (data not shown).

Next, we tested individually all known t-Utps: Utp4, Utp5, Utp8, Utp9, Utp10, Utp15, Utp17, and Pol5 by Ch-IP 
(Fig. 1C-F). Unexpectedly, the level of t-Utp enrichment at the 5 '-ETS position was quite marginal and, for most bait, below or close to the significance threshold. Further, close inspection revealed no significant enrichment at the rDNA promoter (amplicon 6). Strikingly, all UTP-A members, but Pol5 (see below), showed a maximum level of interaction at amplicon 11 that corresponds to the 5 '-end of the $25 \mathrm{~S}$ gene. In parallel, we tested members of other SSUprocessome subcomplexes, including the prototypic Utp1 and Utp22, for UTP-B and UTP-C, respectively. We also

A

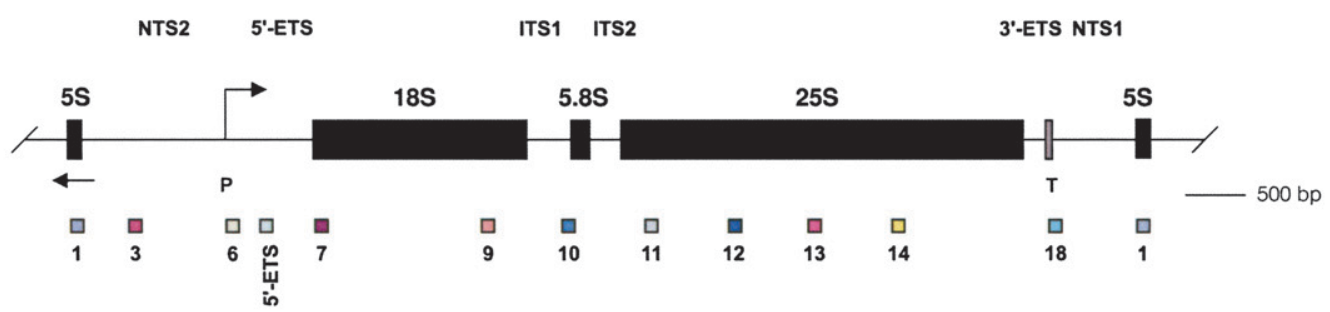

B

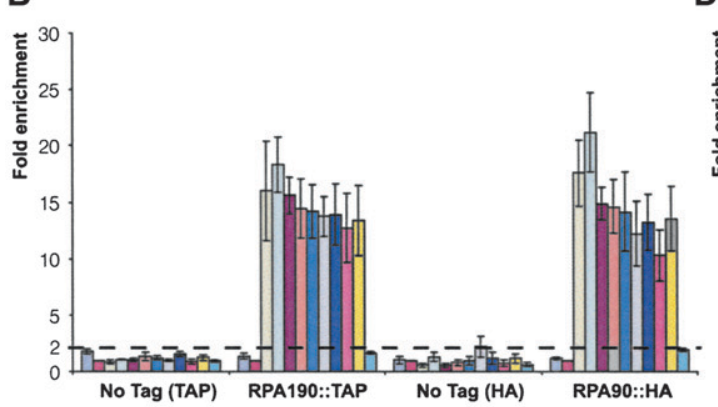

C

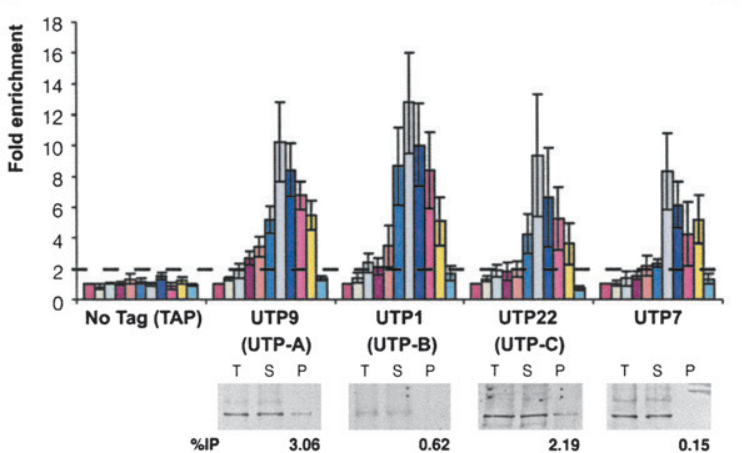

$\mathbf{F}$
D

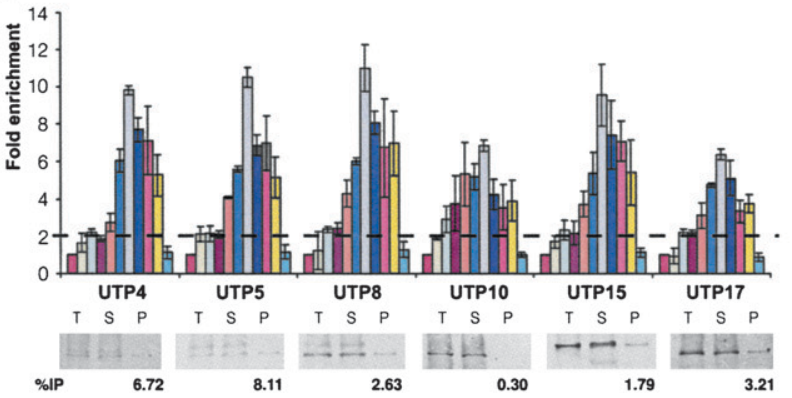

E

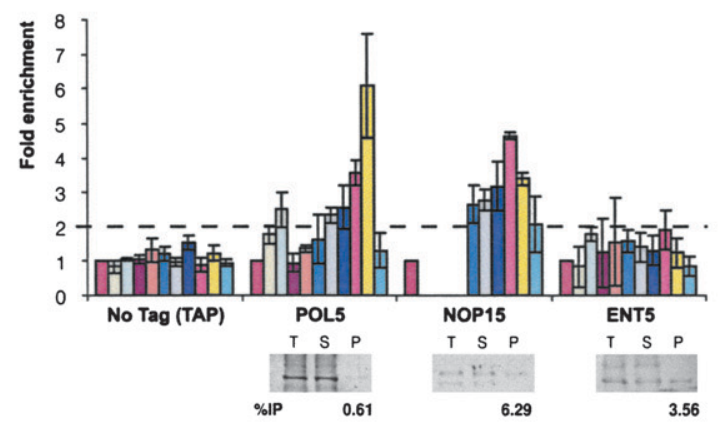

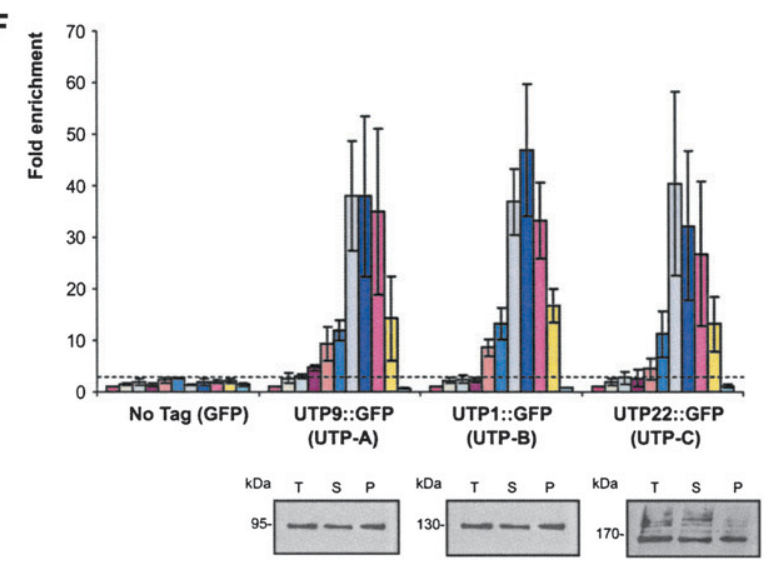

FIGURE 1. (Legend on next page) 
analyzed Utp7, which has not been associated with any of the UTP-A, UTP-B, or UTP-C subcomplexes (Fig. 1C). Interestingly, Utp1, Utp7, and Utp22 all showed a profile of interaction with the rDNA that was virtually undistinguishable from that observed with UTP-A components with a striking peak at amplicon 11 (Fig. 1C). As a control, each bait protein was detected in the chromatin pellet fractions by quantitative Western blot analysis (Fig. 1C-E).

To further consolidate our Ch-IP data set, rDNA interaction profiles were independently established for one component of each of the three known SSU-processome subcomplexes (Utp1, Utp9, and Utp22) in strains that expressed functional carboxyl-terminal GFP fusions (Fig. 1F). The rDNA interaction profiles were similar to those observed with TAP-tagged strains.

To control the specificity of our Ch-IP procedure, an unrelated nucleolar factor required for 605 subunit synthesis (Nop15) (Harnpicharnchai et al. 2001) and a cytoplasmic protein (Ent5) were analyzed (Fig. 1E). Nop15 showed a distinctive rDNA interaction profile, with a strongest interaction mapping roughly to the middle portion of the $25 \mathrm{~S}$ gene (amplicon 13); as expected, the cytoplasmic bait did not show any specific interaction with the rDNA.

Early characterization of UTP subcomplexes indicated that Pol5, so named because it bears similarity to DNA polymerases (Shimizu et al. 2002), is likely a substoichiometric component of UTP-A (Gallagher et al. 2004; Krogan et al. 2004). This was not confirmed in recent affinity purifications (Perez-Fernandez et al. 2007). Interestingly, in our Ch-IP analysis, Pol5 showed a distinctly different pattern of interaction with the rDNA, with a maximal association significantly shifted toward the $3^{\prime}$-end of the rDNA gene, corresponding to amplicon 14 (Fig. 1E). This suggests that Pol5 is unlikely a bona fide core component of UTP-A. Pol5 was previously hypothesized to be involved in Pol I termination (Shimizu et al. 2002); its presence at the vicinity of the terminator would appear compatible with such a possibility.
The interaction of Utps at the $5^{\prime}$-end of the $25 S$ gene indicated a proximity to the rDNA at this position that could either result from a direct interaction, a recruitment by Pol I, or the presence within nascent pre-40S subunits that, in yeast, come in close proximity to the rDNA at that position (see Fig. 8, below; Osheim et al. 2004). Of these, the possibility that the Ch-IP-based assay detects nascent pre-ribosomes seemed likely since the interaction of a $60 \mathrm{~S}$ ribosome synthesis factor was shifted toward the $3^{\prime}$-end of the $25 \mathrm{~S}$ gene, which is exactly what would be expected for the detection of nascent pre-60S subunits on Christmas trees (see Fig. 8, below).

To discriminate between these possibilities, we first tested whether the interaction of Utps at the $5^{\prime}$-end of the $25 \mathrm{~S}$ gene is dependent upon the presence of Pol I. The recruitment of Pol I to the rDNA promoter requires an evolutionarily conserved interaction between its essential subunit Rpa43 and the initiation factor Rrn3 (Peyroche et al. 2000). Transcription was thus inhibited at the initiation level by use of a tet::rpa43 regulatable strain. Following $12 \mathrm{~h}$ of depletion in doxycycline-containing medium, the amount of Pol I throughout the rDNA locus, including at amplicon 11, was indeed substantially reduced (Fig. 2A). In the presence of reduced Pol I, the interaction between Utp4, Utp7, and Utp9 and the rDNA at amplicon 11 was lost (Fig. 2B). As controls, the steady-state level of Utp4, Utp7, and Utp9 was established by quantitative Western blot analysis and was found largely unaffected (Fig. 2B). Interestingly, there was an undocumented and opportune reduction of $\sim 60 \%$ in the steady-state level of Rpa190 in the absence of Rpa43.

To further discriminate whether it is the physical presence of Pol I or that of nascent transcripts that is required for the interaction of SSU-processome components at the $5^{\prime}$-end of the $25 \mathrm{~S}$ gene, chromatin extracts were treated with RNase prior to coprecipitation and qPCR analysis (see Abruzzi et al. 2004). Treating the samples with RNase did not strongly affect the distribution of Pol I within the

FIGURE 1. Components of the SSU-processome colocalize at the $5^{\prime}$-end of the $25 \mathrm{~S}$ gene. (A) Yeast rDNA unit. The Pol I transcript extends from the promoter $(\mathrm{P})$ to the terminator $(\mathrm{T})$ regions of the rDNA and encodes the $18 \mathrm{~S}, 5.8 \mathrm{~S}$, and $25 \mathrm{~S}$ rRNAs. Mature rRNAs are interspersed with external ( $5^{\prime}$ - and $3^{\prime}$-ETS) and internal (ITS1 and ITS2) transcribed spacers. 5S is transcribed by Pol III in the opposite direction. 5S is embedded in nontranscribed regions 1 and 2 (NTS1 and NTS2). Amplicons used in the Ch-IP analysis are indicated (1, 3, 6, 5' -ETS, 7, 9-14, and 18). Scale bar is 500 bp. (B) Distribution of the largest subunit of Pol I (Rpa190) along the rDNA unit. Chromatin extracts prepared from yeast cells expressing either a functional carboxyl-terminal HA or TAP epitope tagged version of Rpa190, and otherwise isogenic wild-type strains (TAP and HA), grown to mid-log phase were submitted to coprecipitation analysis with IgG-coated magnetic beads and the copurifying DNAs specifically analyzed by qPCR with oligonucleotide pairs specific to the entire stretch of the rDNA locus $(A)$. Fold enrichment at each position (color-coded as in panel $A$ ) corresponds to the IP/input ratio normalized to amplicon 3. The threshold of significant enrichment was arbitrarily set to 2 (dashed line). $(C, F)$ Distribution along the rDNA locus of representative UTP proteins from the UTP-A, UTP-B, and UTP-C subcomplexes of the SSUprocessome (Utp1, Utp9, and Utp22), as well as that of unrelated Utp7, expressed either as functional carboxyl-terminal TAP (panel C) or GFP (panel F) epitope tagged constructs. (D) Distribution along the rDNA locus of 6 Utps from the UTP-A subcomplex. (E) Distribution of Pol5 (a suspected UTP-A member), Nop15 (a nucleolar protein involved in 60S ribosome synthesis), and Ent5 (a cytoplasmic protein) along the rDNA locus. ( $C-F$, upper panels) Ch-IP analysis in yeast strains expressing functional carboxyl-terminal TAP- (panels $C-E$ ) or GFP (panel $F$ )-tagged fusion of each bait protein. (Lower panels) Western blot analysis of protein coprecipitation efficiency. Chromatin extracts from the total (T), supernatant $(\mathrm{S})$, and pellet $(\mathrm{P})$ fractions were loaded in a 1:1:10 ratio in SDS-PAGE and analyzed by quantitative Western blot (ECLplex, panels $C-E$ ) or regular ECL (panel $F$ ). In the ECLplex analysis, the value obtained for the T fraction was set to 100 and the IP efficiency expressed as P/T $\times$ 100 ratio. 
transcription unit (data not shown). In these conditions, neither Utp1 (UTP-B) nor Utp9 (UTP-A) interacted specifically at amplicon 11 (Fig. 1C).

In conclusion, pre-40S ribosome synthesis factors were detected in association with the rDNA in an interaction that was at its strongest level at the $5^{\prime}$-end of the $25 \mathrm{~S}$ gene and that required both ongoing transcription and the physical presence of nascent transcripts. This indicates that it is the presence of ribosome synthesis factors within nascent pre-ribosomes that is monitored by Ch-IP.

\section{Involvement of SSU-processome components in RNA synthesis}

t-Utps (UTP-A) were previously involved in efficient Pol I transcription (Gallagher et al. 2004; Prieto and McStay 2007). The conspicuous absence of t-Utps at the rDNA promoter region and the persistent presence in Northern blots and pulse-chase labelings of primary ribosomal transcripts in strains depleted for t-Utp proteins (see Fig. 5, below; Dez et al. 2007; data not shown) led us to reevaluate the proposed function of $\mathrm{t}$-Utps in Pol I transcription by high-resolution transcriptional run-on (TRO) analysis.

First, we established the TRO profiles of two Pol I mutants (Rpa190 and Rpa12). Rpa190 was depleted in a tet:: regulated allele; Rpa12 encodes a nonessential subunit of Pol I involved in transcription termination and was directly obliterated in the genome of haploid yeast cells. Logarithmic yeast cultures were treated with $10 \%$ sarkosyl, which permeabilizes cell membranes and reversibly blocks elongating polymerases (Prescott et al. 2004), incubated with $\left[\alpha^{32} \mathrm{P}\right]$-UTP and transcription allowed to resume for the optimized labeling time of $5 \mathrm{~min}$. Neosynthesized, radiolabeled transcripts were extracted, partially hydrolyzed, and used to probe slot-blots loaded with singlestranded DNA fragments complementary to the entire rDNA locus (Fig. 3). Two types of probes were used: (1) purposely designed antisense rRNA oligonucleotides $(\sim 80$ mer), standardized for their A content (probes A to L), and (2) conventional single-stranded M13 phagemids encoding either specific rDNA regions (probes Pro, 18S, 25S) or a segment of the Actin gene (Act) (Prescott et al. 2004). As expected, depleting Rpa190 strongly impacted pre-rRNA synthesis, as confirmed both in hybridizations with large antisense oligonucleotides or conventional single-stranded phagemids (Fig. 3). We also confirmed that, as expected, deleting Rpa12 led to a substantial level of Pol I terminator read-through (data not shown). Importantly, this initial analysis further indicated that 5S (Pol III, probe L) incorporation was reproducibly very stable, in particular in conditions of Pol I inhibition and much more so than actin labeling (Pol II) (Fig. 3B; Supplemental Table S1); 5 S rRNA incorporation was thus used for normalization in all further experiments (see Fig. 4).
Utp5 and Utp15 (both UTP-A members), as well as Utp7 (which does not belong to any of the three currently described SSU-processome subcomplexes), were selected for detailed analysis and expressed from a regulatable
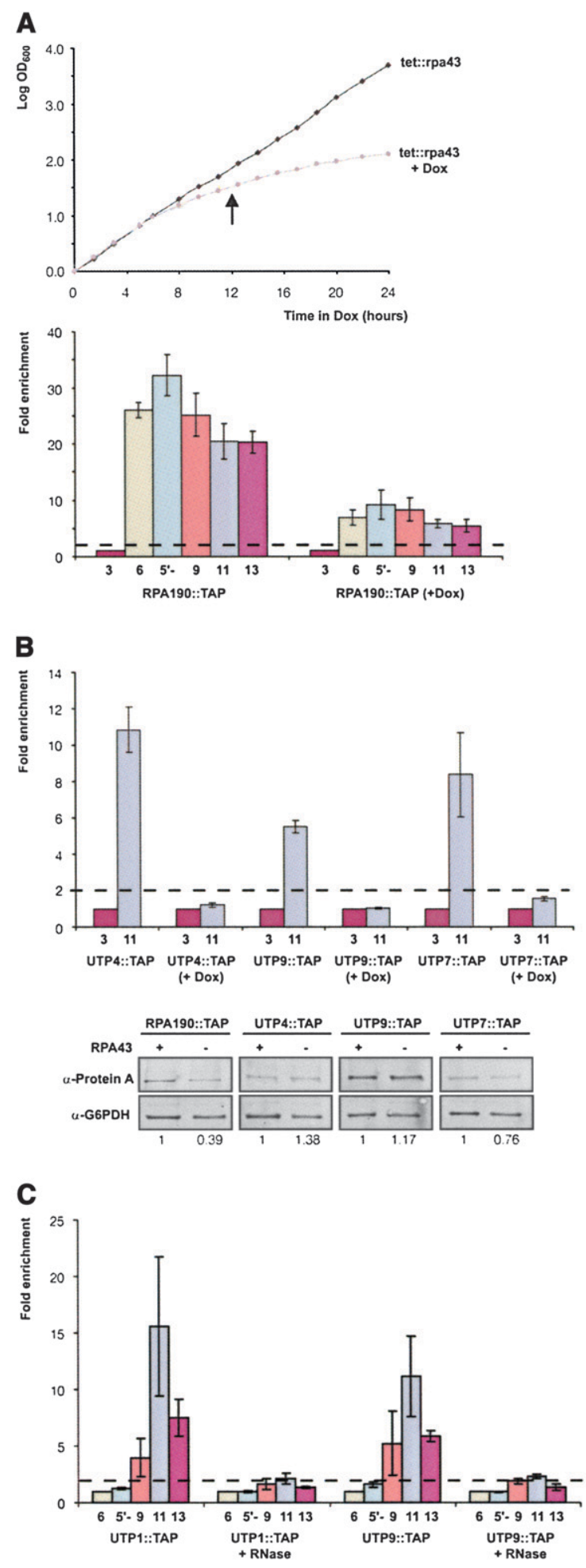

FIGURE 2. (Legend on next page) 
GAL-promoter as amino-terminal HA tagged fusions, the presence of the tag allowing us to establish the residual level of expression of the target protein. Each strain was grown in galactose (permissive condition) and transferred to a glucose-based medium to achieve depletion. At the time points of depletion selected $(3,6$, and $8 \mathrm{~h}$ for Utp7, Utp15, and Utp5, respectively), the cells were still growing efficiently albeit with a much increased doubling time (data not shown). Quantitation established that Utp5 and Utp15 were reduced to $\sim 5 \%$ and $15 \%$ of their physiological level, respectively; Utp7 fell below Western blot detection sensitivity (Fig. 4B). Visual inspection of the slot blots and PhosphorImager quantitation indicated that neither the depletion of Utp5, Utp7, nor Utp15 inhibited pre-rRNA synthesis significantly (Fig. 4C,D).

\section{UTP-A is dispensable for SSU-processome mediated cleavages at sites $A_{0}-A_{2}$ upon surveillance inactivation}

A nucleolar surveillance activity comprised of the nuclear polyadenylation TRAMP complexes acting as nuclear cofactors for the Exosome has been described (see Introduction). In this pathway, "faulty" pre-rRNAs are targeted for selective $3^{\prime} \rightarrow 5^{\prime}$ degradation by the Exosome following binding and/or polyadenylation by TRAMP. How defective

FIGURE 2. Localization of SSU-proccesome components at the $5^{\prime}$ end of the $25 \mathrm{~S}$ gene is dependent upon ongoing transcription and the physical presence of nascent transcripts. (A) Depletion of Rpa43 severely impacts the distribution of Rpa190 along the rDNA. (Upper panel) Growth curves. tet::rpa43 cells were grown in complete medium to mid-log phase $\left(\mathrm{OD}_{600} \sim 0.3\right)$, doxycycline (Dox) was added at a final concentration of $10 \mu \mathrm{g} / \mathrm{mL}$, and growth followed by $\mathrm{OD}_{600}$. $(X$ axis) time in Dox (hours); ( $Y$ axis) $\mathrm{OD}_{600} / \mathrm{OD}_{600}$ at 0 min expressed in a log scale. (Lower panel) Distribution of Rpa190 along the rDNA in the presence and in the absence (+Dox) of Rpa43. Depletion of Rpa43 was achieved following $12 \mathrm{~h}$ of transfer to doxycycline-containing medium (see arrow, panel $A$ ). Levels of Rpa190 interaction with the rDNA at each position were tested as described in Figure 1. Amplicons colorcoded and numbered as in Figure 1. (B) In the presence of reduced recruitment of Pol I, SSU-processome components do not interact at the $5^{\prime}$-end of the 25S gene (amplicon 11). (Upper panel) The presence of Utp4, Utp7, and Utp9 at the $5^{\prime}$-end of the $25 \mathrm{~S}$ gene was tested by Ch-IP in the presence of either physiological (no Rpa43 depletion) or strongly reduced Pol I recruitment (+Dox, corresponding to $12 \mathrm{~h}$ depletion of Rpa43, see panel A). (Lower panel) Effect of Rpa43 depletion on the steady-state level of Rpa190, Utp4, Utp7, and Utp9. Equivalent amounts of chromatin, according to cell $\mathrm{OD}_{600}$, extracted in the presence and absence ( $12 \mathrm{~h}$ depletion time point) of Rpa43, were analyzed by SDS-PAGE and processed for ECLplex. As a control for loading, membranes were probed for G-6-PDH. The amount of target protein was normalized to G-6-PDH and set to 1 in the presence of Rpa43. (C) In the absence of RNA, SSU-processome components do not interact at the $5^{\prime}$-end of the $25 \mathrm{~S}$ gene. Distribution of Utp1 (UTPB) and Utp9 (UTP-A) along the rDNA locus in the presence and in the absence of RNA. Chromatin extracts were treated (+RNase) or not with RNase prior to coprecipitation and qPCR analysis (see Materials and Methods).
pre-rRNAs are recognized by nucle(ol)ar surveillance is, however, currently not understood.

To get initial insight into this selection process, several components of the SSU-processome were depleted and the effect of their absence tested on nucleolar surveillance activation. Since the UTP-A subcomplex has recently been described as an early pre-rRNA binder (see Introduction), we focused our analysis on two UTP-A proteins, Utp5 and Utp15 (Fig. 5; data not shown). As a surrogate, we used Utp11 that does not belong to any of the UTP-A, UTP-B, or UTP-C subcomplexes. TRAMP inactivation was achieved by obliteration of TRF5 in the genome of haploid yeast cells. Exosomal activity was inactivated upon deletion of one of its nuclear cofactor, Rrp6. We primarily targeted Trf5 (and thus TRAMP5) as its deletion does not affect cellular growth allowing direct interpretation of the results, unlike that of its paralog Trf4 (Houseley and Tollervey 2006; Dez et al. 2007; data not shown).

Total RNA was extracted from mid-log phase yeast cells in the presence or in the absence of Utp5, Utp11, or Utp15, separated on denaturing agarose gels, and processed for Northern blot hybridization. Depletion of Utp proteins was here achieved in tetracycline-regulated strains.

Inactivation of TRAMP5 or the Exosome, individually and in combination, led to the stabilization of the 23S RNA (Fig. 5, panels I, lanes 1-4,45-48). The 23S RNA results from a direct cleavage of the $35 \mathrm{~S}$ pre-rRNA at site $\mathrm{A}_{3}$, within ITS1, by RNase MRP in the absence of SSUprocessome mediated cleavages at sites $\mathrm{A}_{0}-\mathrm{A}_{2}$ (see Supplemental Fig. S1). This was particularly striking upon RRP6 deletion (Fig. 5, panels I, lanes 3,47; Allmang et al. 2000). As expected for SSU-processome components, depletion of Utp5 (Fig. 5, panels I, lanes 5-9), Utp11 (Fig. 5, panels I, lanes 25-29), or Utp15 (data not shown) led to the inhibition of pre-rRNA processing at sites $\mathrm{A}_{0}-\mathrm{A}_{2}$ with the consequent disappearance of the $33 \mathrm{~S} / 32 \mathrm{~S}$ (Fig. 5, panel I, Utp5, panels I-IV, Utp11), 27SA 2 (Fig. 5, panels II), and $20 \mathrm{~S}$ prerRNAs (Fig. 5, panels I), and failure to synthesize $18 \mathrm{~S}$ rRNA (Fig. 5, panels III).

Depleting Utp5 in cells inactivated for TRAMP5 (Fig. 5, panels I,II, lanes 10-14) led to a striking stabilization of the 23S RNA, a marginal $20 \mathrm{~S}$ pre-rRNA restoration (Fig. 5, panel I) and a partial, but reproducibly significant, restoration in $18 \mathrm{~S}$ rRNA (Fig. 5, panel III). Inactivation of the Exosome (Fig. 5, lanes 15-19) led to a further stabilization of the 23S RNA, a noticeable stabilization of the $20 \mathrm{~S}$ prerRNA and an increased restoration in $18 \mathrm{~S}$ rRNA. Strikingly, inactivating both TRAMP5 and the Exosome in cells deprived of Utp5 (Fig. 5, lanes 20-24) had synergistic effects on 23S RNA stabilization, and on 20S pre-rRNA and $18 \mathrm{~S}$ rRNA accumulation. An identical analysis with another UTP-A member, Utp15, led to overall similar conclusions (data not shown). Globally, inactivating TRAMP5 or the Exosome in cells deprived of Utp11 had quite a similar effect on 23S RNA stabilization (Fig. 5, panels I,II, lanes 
A

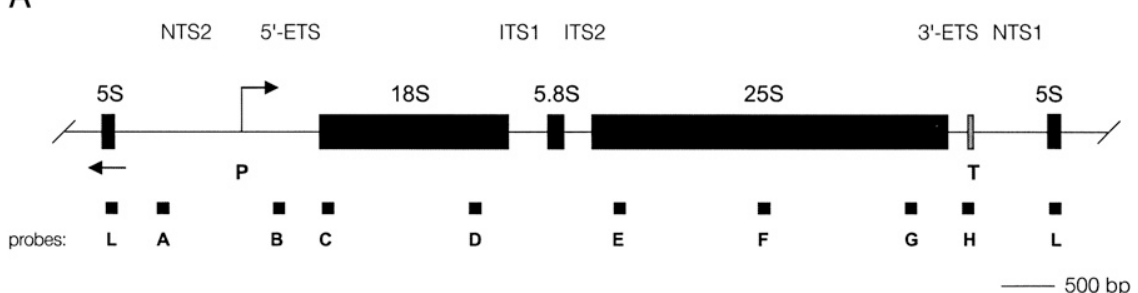

B

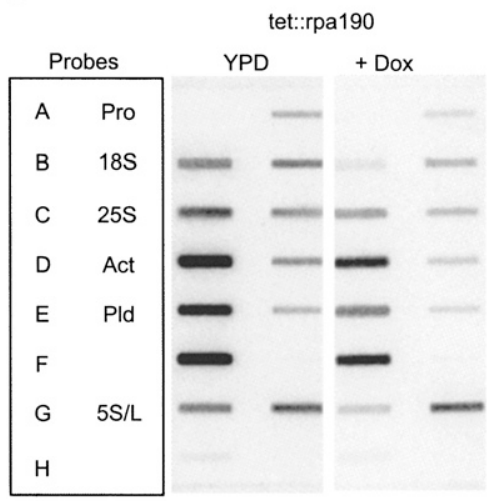

C

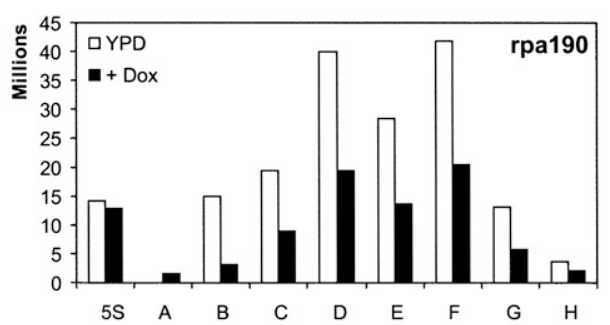

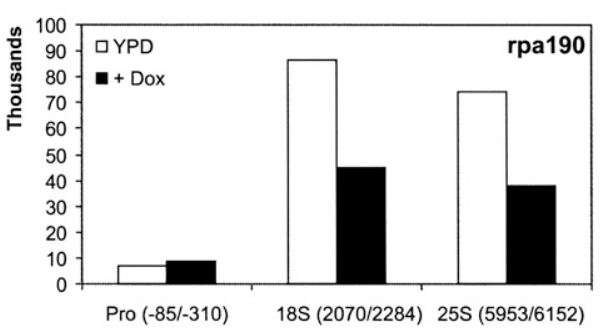

FIGURE 3. High-resolution transcriptional run-on (TRO) analysis of Rpa190 depletion. $(A)$ Yeast rDNA unit. See Figure 1 for description. Probes (A-L) used in the TRO analysis are indicated. (B) Rpa190 depletion strongly impacts rRNA synthesis. TRO was performed in tet::rpa190 and otherwise isogenic wild-type cells grown to mid-log phase in complete medium in the presence or absence of doxycycline (+Dox). Depletion of Rpa190 was achieved for $6 \mathrm{~h}$. Each labeling was performed independently in triplicate; a representative example is shown. Slot blots were loaded with antisense oligonucleotides normalized for their A content (probes A to L) and, for comparison, with conventional single-stranded phagemids, encoding portions of the rDNA (probes Pro, 18S, 25S), the Actin gene (Act), or without insert, providing background hybridization (Pld) (see Materials and Methods). (C) Results presented in panel $B$ were quantitated with a PhosphorImager (Typhoon 9200, GE Healthcare). (Top panel) TRO analysis with oligonucleotides, and (bottom panel) with phagemids (rRNA regions targeted in the hybridizations are indicated).
SSU-processome, but not in strains deprived of either of two unrelated Utps (Utp11 and Utp20), points to a "classspecific" rather than a "protein-specific" role for UTP-A in ribosome synthesis quality control mechanisms.

In conclusion, depleting individual constituents of the SSU-processome, regardless of whether they belong to the UTP-A subgroup or not, disabled its processing activity in cleavage at sites $\mathrm{A}_{0}-\mathrm{A}_{2}$, allowing the RNase MRP to operate first in cleaving pre-rRNAs at site $\mathrm{A}_{3}$ and generating the 23S RNA. Under physiological conditions, the nucleolar surveillance is active and the 23S RNA rapidly identified by TRAMP and degraded by the Exosome, in agreement with Dez et al. (2007). Partial (deletion of TRF5 or RRP6) or complete inactivation of the surveillance (simultaneous deletion of TRF5 and RRP6) led to the accumulation of the 23S RNA. Strikingly upon depletion of a UTP-A component (Utp5 or Utp15), the 23S RNA was quantitatively matured into $20 \mathrm{~S}$ pre-rRNA and $18 \mathrm{~S}$ rRNA; this was not the case upon depletion of a non UTP-A protein (Utp11). Significantly, this indicates that UTP-A, a primary pre-rRNA binder, can be discriminated functionally from other SSU-processome subcomplexes in that its requirement in cleavages at sites $A_{0}-A_{2}$ can specifically be overcome in conditions of surveillance inactivation.
30-44); however, the restoration of $20 \mathrm{~S}$ pre-rRNA was much less pronounced for Utp11 than for Utp5, and it was not accompanied by a significant restoration in $18 \mathrm{~S}$ rRNA (Fig. 5, panel III, cf. lanes 20-24 and 40-44). Consistent with our analysis, the 23S RNA was stabilized in cells depleted for the UTP-A protein Utp10 or the unrelated Utp20 and inactivated for Trf5, with a modest but specific increase in $20 \mathrm{~S}$ pre-rRNA for Utp10 (Dez et al. 2007). The effects on mature rRNA restoration largely escaped previous analysis since it required the simultaneous inactivation of Trf5 and Rrp6 to be seen. Altogether, the consistent observations that, in the absence of functional surveillance, the levels of physiological pre-rRNA intermediates and/or mature rRNA were either increased or restored in strains depleted for Utp5, Utp10, and Utp15, corresponding to three out of six members of the UTP-A subcomplex of the

\section{Pre-rRNAs accumulated in the absence of SSU-processome components are polyadenylated by TRAMP5}

Previous work established that RNA degradation by the Exosome is in part promoted by recognition and/or polyadenylation of its substrates by TRAMP (see Introduction; for reviews, see Houseley et al. 2006; Andersen et al. 2008; Lebreton and Seraphin 2008). What we wanted to address here was whether pre-ribosome assembly defects, consecutive to depletion of Utp proteins, would be identified by nucleolar surveillance and whether it would stimulate pre-rRNA polyadenylation by TRAMP.

To test this idea directly, the poly(A) status of pre-rRNAs stabilized in Exosome deficient strains upon depletion of Utp5 or Utp11 was established by affinity purification 
A

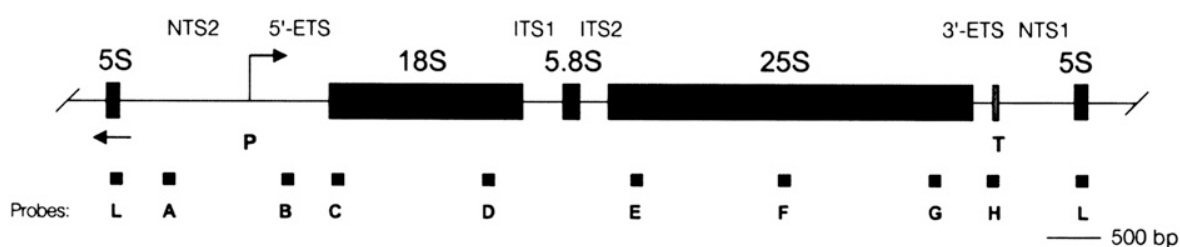

B
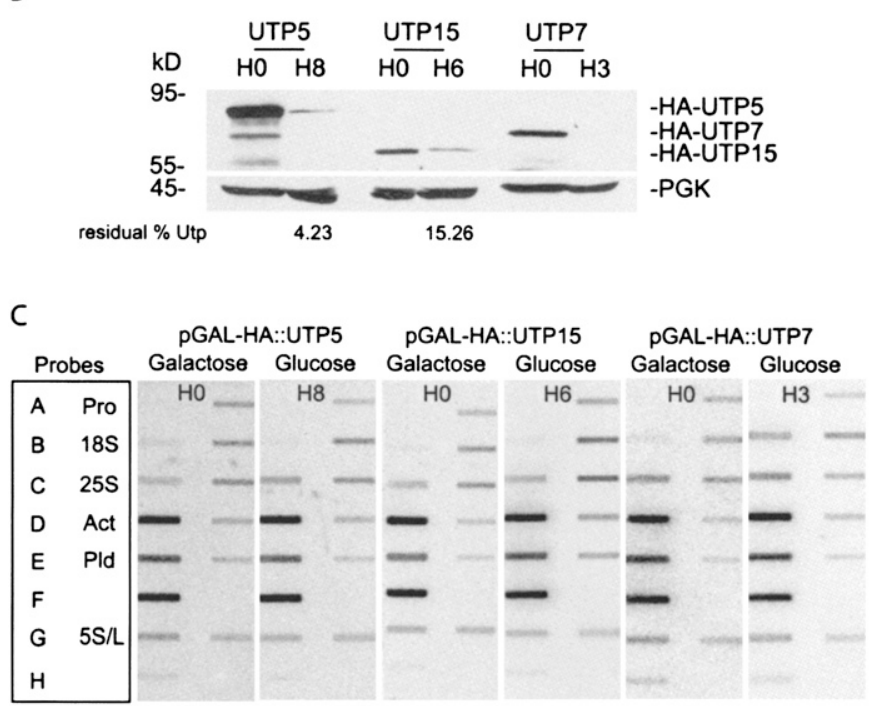

D
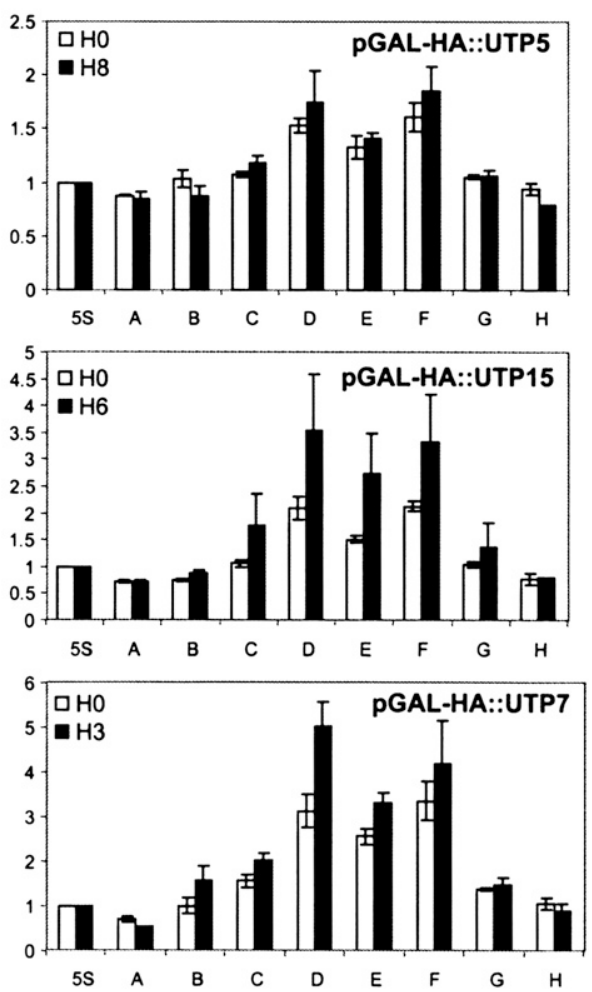

FIGURE 4. Involvement of SSU-processome components in rRNA synthesis. (A) rDNA unit and probes used in the TRO analysis, as described in Figure 3. (B) Residual level of expression of targeted Utps (Utp5, Utp7, and Utp15) in GAL-regulated strains expressing functional aminoterminal HA epitope tagged constructs. Yeast cells were grown to mid-log phase in galactose medium and transferred to glucose for the indicated time points. Equivalent amounts of total protein extracts were analyzed by $\alpha$-HA Western blotting. PGK was used as a loading control. Residual level of expression of Utp5 and Utp15 were quantitated with ImageQuant (GE Healthcare). (C) TRO analysis was conducted as described in Figure 3. The experiments were performed in triplicate, and representative slot blots are shown. $(D)$ PhosphorImager quantitation. Signal was normalized to $5 \mathrm{~S}$.

using oligo-dT selection (Fig. 6; Supplemental Fig. S2). First, analysis of total RNA confirmed that in the absence of functional nucleolar surveillance (simultaneous deletion of TRF5 and RRP6), 20S pre-rRNA accumulation was only specifically restored upon depletion of a UTP-A member (Fig. 6, cf. lanes 6,7 in panels III in Fig. 6, Supplemental Fig. S2). Deletion of RRP6 led to the accumulation of elevated levels of polyadenylated pre-rRNAs that were essentially all lost upon further deletion of TRF5 (Fig. 6, lanes 8-10; Kuai et al. 2004; LaCava et al. 2005; Wyers et al. 2005; Houseley and Tollervey 2006). In the absence of Exosomal activity, elevated levels of polyadenylated pre-rRNAs were also observed upon depletion of SSU-processome components
(Fig. 6, lanes 11,12; Supplemental Fig. S2, lanes 17,18) and, similarly, these were lost upon further inactivation of Trf5 (Fig. 6, lanes 13,14; Supplemental Fig. S2, lanes 15,16). Further, since Trf4, the paralog protein to Trf5, is unaffected in these cells, we conclude that it is TRAMP5 that carries the bulk of this polyadenylation activity (see Houseley and Tollervey 2006). Significantly, the accumulation of polyadenylated 23S RNA was substantially increased both in the absence of Utp5 (Fig. 6, lanes 11,12, best seen in panel I) and Utp11 (Supplemental Fig. S2, lanes 17,18), indicating that 23S RNA accumulated in this mutant context are readily tagged for destruction by TRAMP-mediated polyadenylation. 


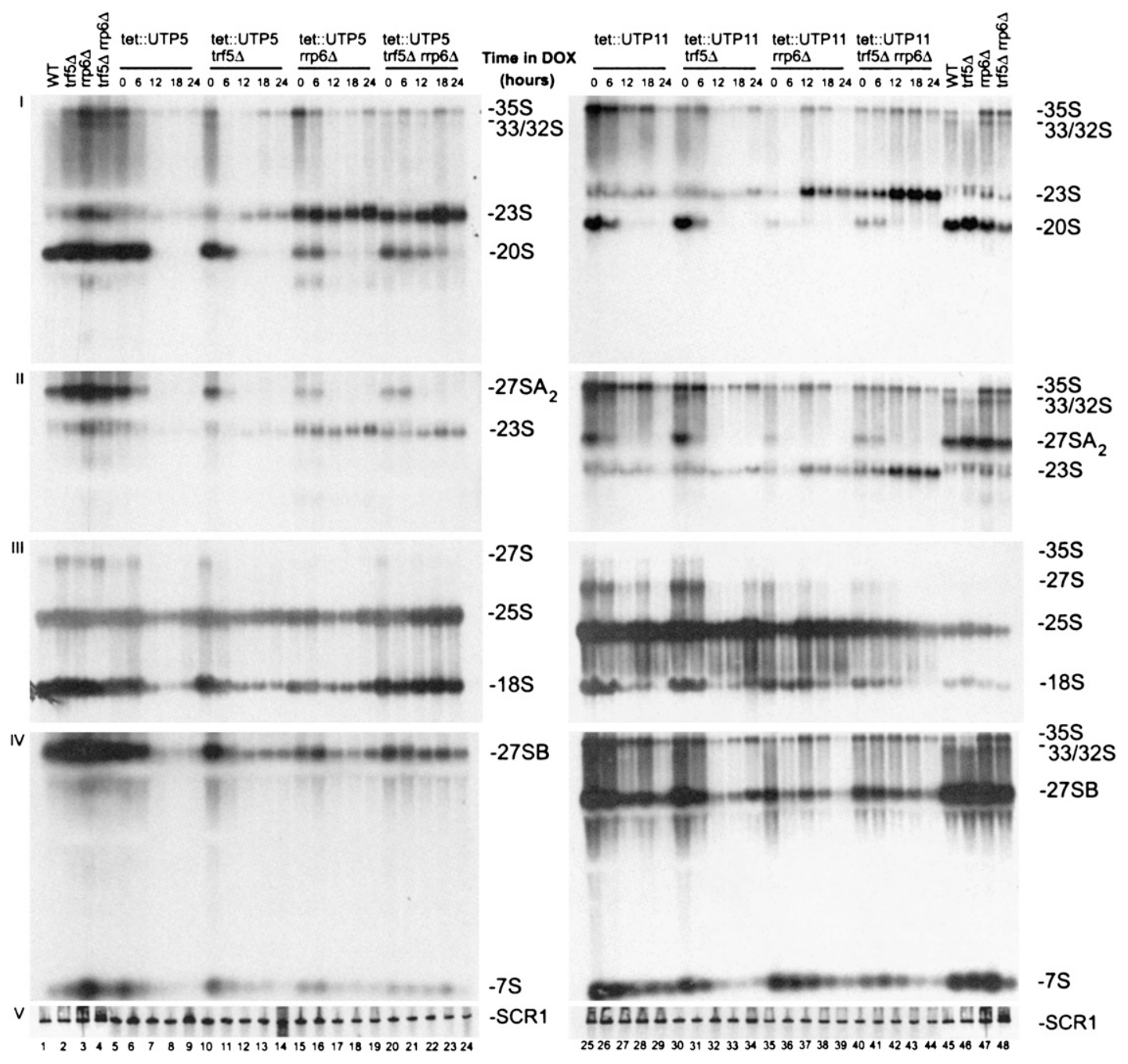

FIGURE 5. Pre-rRNA and rRNA are stabilized upon TRAMP and Exosome inactivation in strains depleted for SSU-processome components. Total RNA was extracted from cells grown to mid-log phase in complete medium, separated in denaturing agarose gels, and transferred to nylon membranes for Northern blot hybridizations. RNA was extracted from an otherwise isogenic wild-type strain, from strains deleted either for the TRAMP component Trf5, the Exosome cofactor Rrp6, or for both (lanes 1-4,45-48), as well as from cells depleted for Utp5 (lanes 5-24) or Utp11 (lanes 25-44), either in the presence or in the absence of functional TRAMP and/or Exosome complexes. UTP depletion was achieved in tet:: regulated strains following transfer to doxycycline-containing medium for the time points indicated (6-24 h). A description of the pre-rRNA processing pathway in yeast, as well as the oligonucleotide probes used in the hybridizations, is provided in Supplemental Fig. S1. Panel I was hybridized with probe b; panel II with c, panel III with a and f, and panel IV with e. As a loading control, membranes were hybridized for SCR1, the RNA component of SRP (panel V).

\section{The nuclear poly(A) polymerase Trf5 colocalizes with nascent pre-ribosomes}

To test whether nucleolar surveillance initiates cotranscriptionally, that is whether misassembled pre-rRNPs are identified concomitantly with RNA synthesis, we established the rDNA interaction profile of TRAMP and Exosome constituents by Ch-IP. Several TRAMP components (Air1, Air2, Mtr4, Trf4, and Trf5) and two catalytic Exosome subunits (Rrp44 and Rrp6) were tested (Fig. 7A). Both Trf4 and Trf5 were significantly coenriched across the rDNA transcriptional unit with a maximum level of interaction at amplicon 11. The Trf4 and Trf5 profiles persisted toward the $3^{\prime}$-end of the gene and were thus not strictly identical to those observed with SSU-processome components. This distribution is compatible with the involvement of TRAMP4 in a proofreading activity of later steps in the ribosome assembly pathway (Dez et al. 2006). Air1 and Air2 also interacted significantly with the rDNA unit. Strikingly, no significant enrichment was observed for the helicase Dob1/Mtr4 or for the catalytic Exosome subunits Rrp6 and Rrp44, suggesting that their interaction may be transient. For Trf5, we further established that the interaction is dependent upon both ongoing transcription and the presence of nascent transcripts, as described above for SSU-processome components (Fig. 7B,C). 


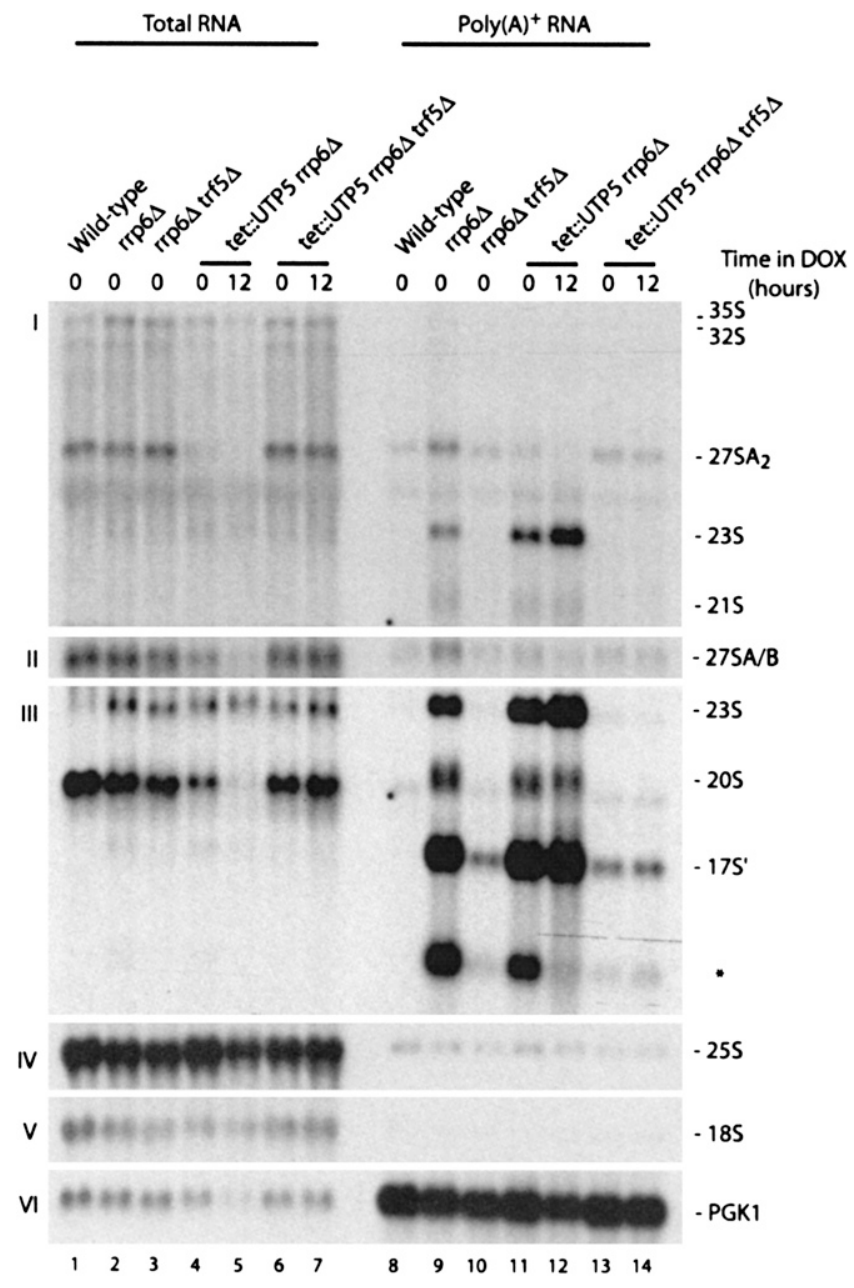

FIGURE 6. Pre-rRNAs accumulated in the absence of Utp5 are polyadenylated by Trf5. Total RNA was extracted from yeast strains grown to mid-log phase in complete medium and subjected to poly $(\mathrm{A})^{+}$affinity purification on oligo-dT coated beads. An otherwise isogenic wild-type strain, strains deleted for the Exosome cofactor Rrp6, or for Rrp6 and the TRAMP component Trf5, either in the presence $(0$ $\mathrm{h}$ time points) or in the absence of Utp5 ( $12 \mathrm{~h}$ depletion time points). Total RNA and purified poly $(\mathrm{A})^{+}$were loaded on a $1.2 \%$ agarose/ formaldehyde gel in a 1:50 ratio. Northern blot hybridization with probes to pre-rRNA, mature rRNA, or the loading control PGK1, a highly expressed messenger RNA, the level of which is largely unaffected by Exosome mutations. Panel I was hybridized with oligo c; panel II with LD906; panel III with b; panel IV with f; panel V with a and panel VI with LD1142 (PGK1). A significant amount of the aberrant 17S' RNA that extends from within the $5^{\prime}$ end of the $18 \mathrm{~S}$ rRNA to $\mathrm{A}_{3}$ (Supplemental Fig. S1; Houseley and Tollervey 2006) remained polyadenylated in the absence of Trf5. It is currently not understood why the RNA species marked with an asterisk, and representing an as yet uncharacterized pre-rRNA degradation product (Houseley and Tollervey 2006), was not coprecipitated upon Utp5 depletion.

\section{DISCUSSION}

\section{Christmas trees visualization by chromatin immunoprecipitation}

We have described a Ch-IP-based approach to follow the cotranscriptional assembly of pre-ribosomes. In this, $40 \mathrm{~S}$ ribosome synthesis factors were found closely associated with the rDNA throughout the Pol I transcription unit with a striking culmination at the $5^{\prime}$-end of the $25 \mathrm{~S}$ gene (amplicon 11; Fig. 1), in an interaction that required both ongoing transcription and the presence of nascent transcripts (Fig. 2). The strong interaction at amplicon 11 correlates with the detection by EM on Chromatin spread of large $5^{\prime}$-terminal SSU-processomes in the vicinity of the rDNA at this position (Fig. 8). UTP-A, UTP-B, and UTP-C were all coenriched at the $5^{\prime}$-end of the $25 \mathrm{~S}$ gene, indicating that, at this position, all three UTP subcomplexes were successfully incorporated into nascent pre- $40 \mathrm{~S}$ ribosomes. We conclude that this Ch-IP data set offers a validation in vivo of the morphological description of the Christmas trees. Accordingly, a $60 \mathrm{~S}$ ribosome synthesis factor interacted strongly with the rDNA at the middle third of the $25 \mathrm{~S}$ gene at a position where the $5^{\prime}$-terminal knobs interpreted as maturing large subunits are detected initially on the rDNA by EM (Fig. 8).

\section{Involvement of SSU-processome components in RNA synthesis}

Owing to their physical proximity to the rDNA (Fig. 1), SSU-processome components offer ideal candidates to interface pre-rRNA synthesis and pre-rRNA processing processes. We have examined the function of three SSUprocessome components (the UTP-A proteins Utp5 and Utp15 as well the unrelated Utp7) in ribosomal RNA synthesis by high resolution TRO analysis and concluded that neither is essential to this process (Fig. 4). A previous report, in yeast that tested several UTP-A components (including Utp5 and Utp15) concluded that t-Utps facilitate optimal and efficient rRNA transcription (Gallagher et al. 2004). In an independent study, metabolic labeling in yeast cells depleted for the UTP-A protein Utp10 or the non-UTP-A Utp20, however, revealed robust ongoing transcription under conditions that greatly impaired pre-rRNA processing (Dez et al. 2007). As discussed below, we believe that the requirement for SSU-processome components in pre-rRNA accumulation lies with their involvement in pre-rRNA turnover rather than RNA synthesis (Figs. 5, 6). Strikingly, several but not all human orthologs to UTP-A proteins were also involved in pre-rRNA synthesis, suggesting that in higher eukaryotes several UTP-A proteins may evolved specific functions (Prieto and McStay 2007).

\section{Early ribosome assembly defects activate nucleolar surveillance}

How and where misassembled pre-rRNPs are identified by surveillance and targeted for degradation remain largely unknown. We have depleted individual components of the SSU-processome (Utp5, Utp15, and Utp11) and tested how pre-ribosomes generated in their absence are targeted by 
A

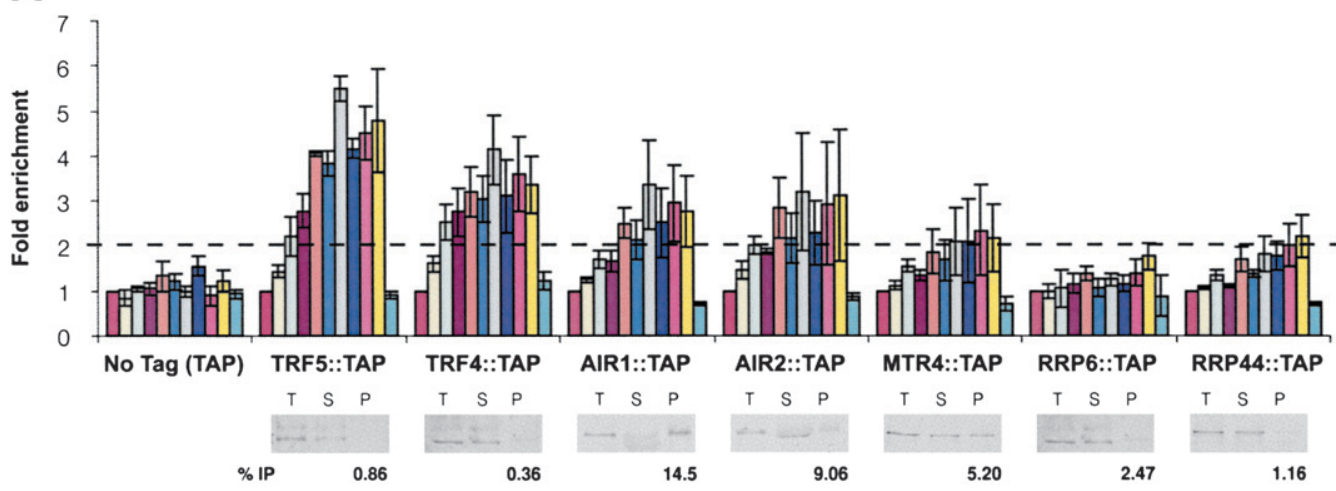

B

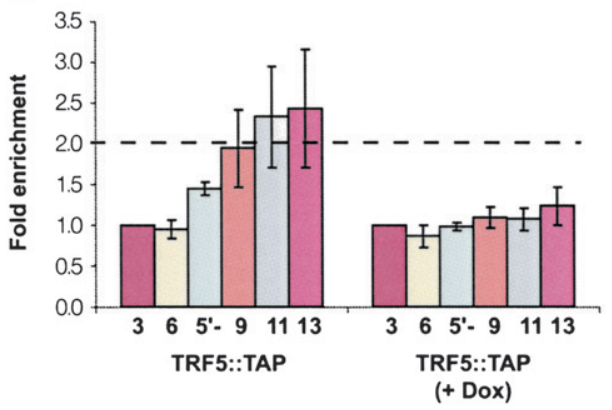

C
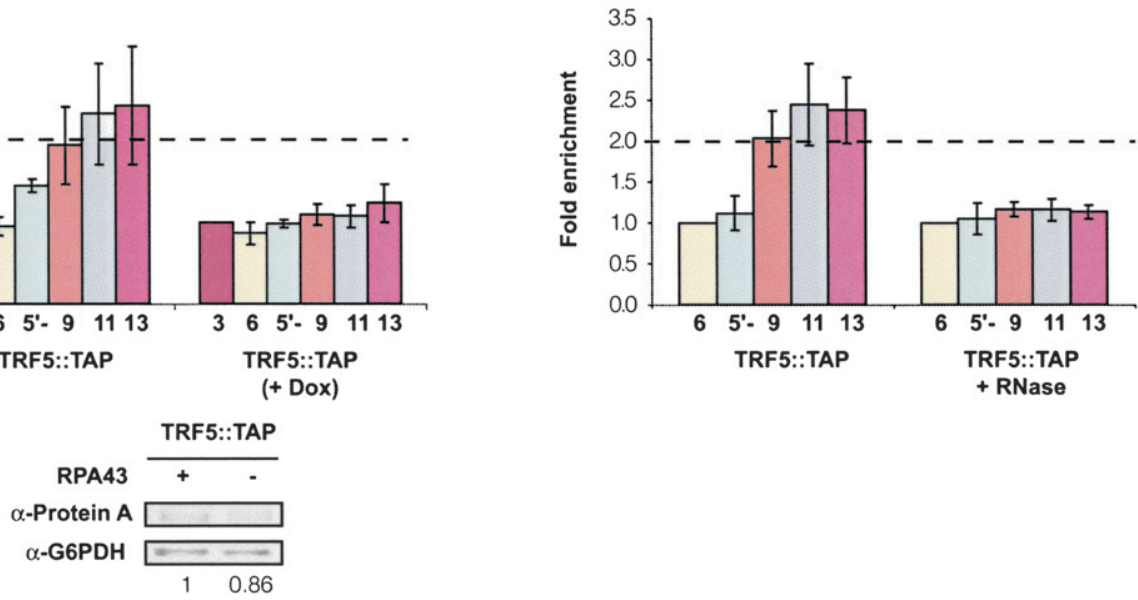

FIGURE 7. Several components of the nucleolar surveillance co-localize with nascent pre-ribosomes. (A) rDNA Ch-IP analysis of components of the TRAMP (Air1, Air2, Trf4,Trf5, and Mtr4) and Exosome (Rrp44 and Rrp6) complexes. (Upper panel) Yeast strains expressing a functional carboxyl-terminal TAP-tagged fusion of Air1, Air2, Mtr4, Rrp6, Rrp44, Trf4, and Trf5 and wild-type isogenic control (TAP) were analyzed by ChIP and qPCR as described in Figure 1. (Lower panel) Western blot analysis of protein coprecipitation efficiency (see legend to Figure 1). $(B$, upper panel) Trf5 is recruited cotranscriptionnally to the rDNA. Ch-IP analysis was performed in the presence and in the absence of Rpa43 (+Dox, $12 \mathrm{~h}$ depletion) (see Fig. 2B). (Lower panel) Effect of Rpa43 depletion on the steady-state level of Trf5 (see Fig. 2B). The depletion of Rpa43 resulted in a mild reduction in the steady-state level of Trf5 of about $15 \%$, the concurrent decrease in the level of interaction with the rDNA was, however, twofold. $(C)$ In the absence of RNA, Trf5 does not interact at the $5^{\prime}$-end of the $25 \mathrm{~S}$ gene. Chromatin extracts were treated (+RNase) or not with RNase prior to coprecipitation and qPCR analysis (see Fig. 2C).

quality control mechanisms. Utp5 and Utp15 both belong to UTP-A, a subcomplex of UTPs recently described as a primary pre-rRNA binder (Gallagher et al. 2004; PerezFernandez et al. 2007). Utp11 does not belong to any of the UTP-A, UTP-B, or UTP-C subcomplexes.

As expected, depletion of SSU-processome components led to pre-rRNA processing inhibitions at cleavage sites $\mathrm{A}_{0}$ $A_{2}$, cleavage of the pre-rRNAs at site $A_{3}$ by RNase MRP, and production of the 23S RNA. Under physiological conditions, and in agreement with earlier observations (Allmang et al. 2000), the 23S RNA was rapidly targeted for selective degradation by surveillance. This involved its recognition and polyadenylation by Trf5 followed by its $3^{\prime}-5^{\prime}$ degradation by Rrp6. Indeed, inactivation of either Trf5 or Rrp6 led to a striking 23S RNA stabilization, and the simultaneous inactivation of both TRAMP5 and the Exosome had synergistic effects. In the absence of Trf5 most polyadenylated RNAs were lost, indicating that it is TRAMP5 that carries the bulk of this activity. Strikingly, inactivating Trf4, rather than Trf5, in cells depleted for either Utp5, Utp11, or Utp15 did not stabilize quantitatively the 23S RNA (data not shown). This is consistent with the proposal that TRAMP5 (pre-40S synthesis) acts earlier than TRAMP4 (pre-60S) in the ribosome synthesis pathway (Dez et al. 2007).

The lack of any obvious protein motifs in many ribosome synthesis factors entails that unraveling their precise functions will await the determination of the three-dimensional structure of pre-ribosomes. In the meantime, the description of preassembled ribosome synthesis factor subcomplexes, such as UTP-A, UTP-B, and UTP-C, and their structural and functional discrimination appears increasingly important. While we could not confirm the previously suggested 


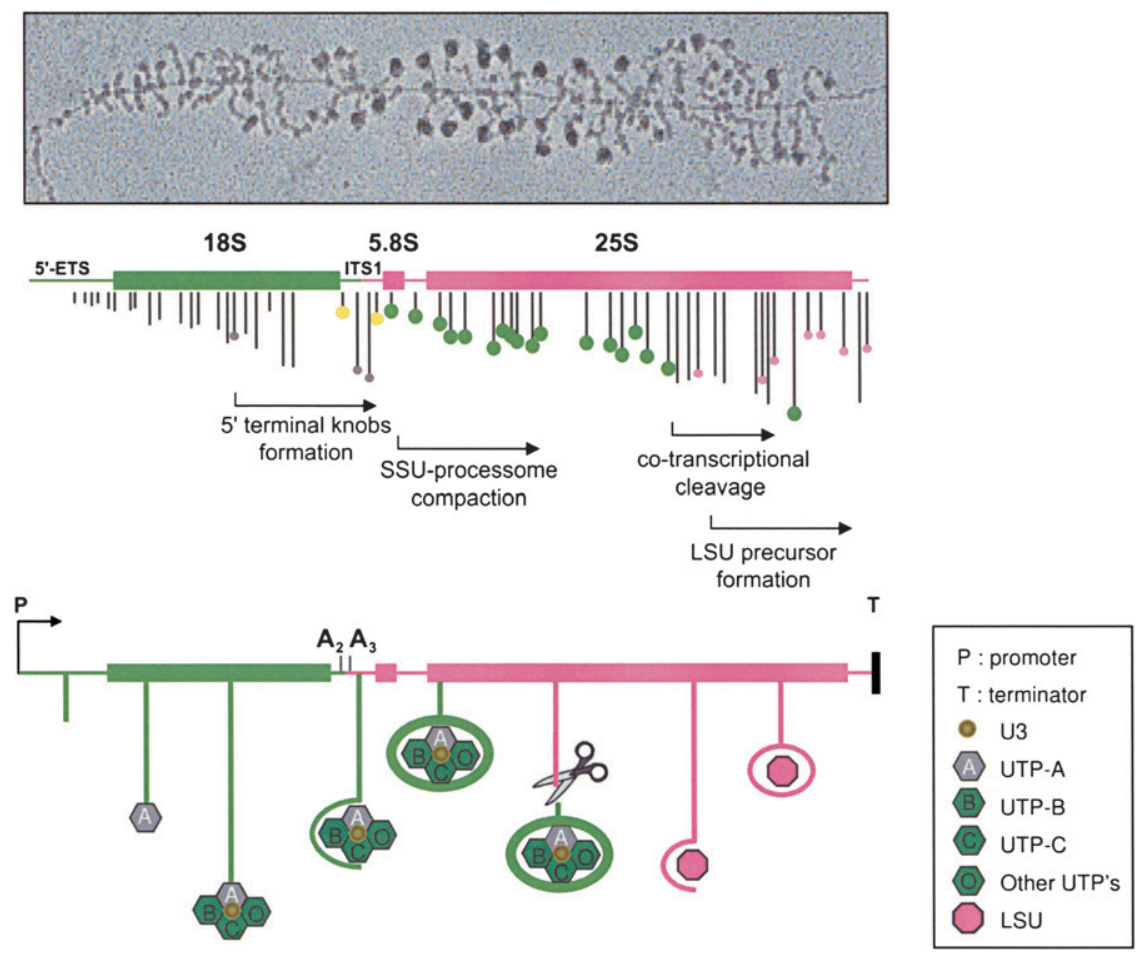

FIGURE 8. SSU-processome assembly pathway. (Upper panel) EM visualization of an actively transcribed rDNA gene by chromatin spread (courtesy of Ann Beyer and Yvonne Osheim, University of Virginia). (Middle panel) Interpretative tracing and transcript mapping (redrawn from Osheim et al. 2004). rDNA is color-coded as follows: $5^{\prime}$-end to center of ITS1 in green; center of ITS1 to the $3^{\prime}$-end in pink. Particles that appear on the transcripts are shown on the tracing as follows: gray particles correspond to the initial small $5^{\prime}$-terminal knobs, yellow to the newly formed (loose) large SSU-processomes, green to the mature (tight) SSU-processomes, and pink to pre-large-subunit knobs that form at the $5^{\prime}$-end of cleaved transcripts. (Lower panel) SSU-processome assembly starts with the binding of the UTP-A subcomplex to nascent transcripts generating a $35 \mathrm{~S} \cdot \mathrm{UTP}-\mathrm{A}$ intermediate. This is a prerequisite step that impinges on the recruitment and assembly of several other UTP subcomplexes (UTP-B, UTP-C, and UTPO). Utps that do not belong to either of the UTP-A, UTP-B, or UTP-C subcomplexes are here tentatively referred to as UTP-O, for Others. The SSU-processome condenses and comes in close proximity to the $5^{\prime}$ end of the $25 \mathrm{~S}$ gene. This correlates with the strongest Ch-IP interaction at amplicon 11 . In a currently estimated $50 \%$ of the cases, this is followed by cotranscriptional cleavage within ITS1, pre-40S subunits release, and large subunit rRNP assembly. Key to the right. (LSU) Large subunit.

involvement of UTP-A (t-UTPs) in transcription initiation (see above), our work brings a novel functional discrimination to that subclass of SSU-processome components in showing that the 23S RNA accumulated in UTP-A defective mutants (Utp5 and Utp15) under inactivation of nucleolar surveillance (inactivation of both Trf5 and Rrp6) was quantitatively matured into $20 \mathrm{~S}$ pre-rRNA and mature $18 \mathrm{~S}$ rRNA. Strikingly, this was not the case upon depletion of the non-UTP-A protein Utp11. We conclude that the requirement for UTP-A members in cleavages at sites $\mathrm{A}_{0}-$ $\mathrm{A}_{2}$ can specifically be bypassed in conditions of nucleolar surveillance inactivation. Interestingly, cells inactivated for nucleolar surveillance and depleted for UTP-A were not viable (data not shown). This indicates that restoring $18 \mathrm{~S}$ rRNA synthesis to near wild-type levels was insufficient to produce $40 \mathrm{~S}$ subunits that could perform their essential function in translation, presumably owing to subtle assembly defects, and further emphasizes the importance of ribosome synthesis quality controls in cell homeostasis.

Finally, we have localized in close vicinity to the Pol I rDNA transcription unit the TRAMP complex components Air1, Air2, as well as the poly(A) polymerases Trf4 and Trf5 (Fig. 7). Trf4 had previously been localized to rDNA intergenic spacer regions, where it is involved in surveillance of Pol II cryptic transcripts (Houseley et al. 2007). The detection of the zinc knuckle and putative RNA binding proteins Airl and Air2 at the rDNA is compatible with their presumed function in recruiting the TRAMP complexes to the RNAs and further suggests that the nucleolar surveillance machinery operates, at least partially, cotranscriptionally. Further, the enrichment of Trf5 at the rDNA, which required both ongoing transcription and the presence of the RNA, also strongly favors cotranscriptional recruitment.

\section{A nucleolar surveillance pathway}

The quality of an RNA is likely to be, at least partly, monitored by its ability to engage in the reactions or processes "which it has evolved to be part of" (Andersen et al. 2008), that of a precursor RNA by its propensity to be part of a streamlined assembly pathway. We have reported that failure to assemble individual SSU-processome components with proper kinetics inhibits early prerRNA processing reactions $\left(A_{0}-A_{2}\right)$, and that, despite the activation of a cryptic alternative processing pathway (cleavage at site $A_{3}$ by MRP) the 23S RNA generated is rapidly targeted by nucleolar surveillance by TRAMP for its selective degradation by the Exosome. This quality control system appeared particularly acute upon depletion of primary prerRNA binders, such as components of the UTP-A subcomplex, since normal cleavage $\left(A_{0}-A_{2}\right)$ was here shown to be not strictly dependent upon their presence.

\section{MATERIALS AND METHODS}

\section{Yeast genetics}

Yeast cells were grown according to standard procedures in YPD ( $2 \%$ peptone, $1 \%$ yeast extract, $2 \%$ glucose). To achieve depletion 
of target proteins, suitable regulatable strains were either transferred from galactose to glucose-based medium (GAL-regulated) or doxycycline (Sigma) added to a final concentration of $10 \mu \mathrm{g} /$ $\mathrm{mL}$. Cultures were kept in mid-log phase at all times by continuous dilution with fresh medium. Yeast strains are listed in Supplemental Fig. Table S2.

\section{Chromatin Immunoprecipitation}

Ch-IP was performed as described by Kwapisz et al. (2008) (see Supplementary Materials and Methods). All experiments were performed in triplicate. DNA material in the IP and Input preparations was quantified by real-time PCR ( $\mathrm{qPCR}$ ) using the Platinum SYBR Green qPCR SuperMix-UDG with Rox (Invitrogen) on Applied Biosystems hardware. Oligonucleotide pairs used are listed in Supplemental Table S4; the position of the amplicons is depicted in Figure 1.

\section{Western blotting}

Western blotting was performed on chromatin fractions using the ECLplex Western blotting system (GE Healthcare Biosciences) or WestPico (Pierce).

\section{Transcriptional run-on analysis}

TRO was performed as described by Prescott et al. (2004). Slot blots were loaded with either single-stranded 80-mers DNA oligonucleotides normalized for their " $A$ " content (see Supplemental Table S5) or conventional single-stranded phagemids.

\section{RNA extraction and Northern blotting}

RNA extraction and Northern blotting were as described previously by Colau et al. (2004). Oligonucleotides depicted in Supplemental Figure S1 are listed in Supplemental Table S6.

\section{$\operatorname{Poly}(\mathrm{A})^{+}$RNA purification}

Poly $(\mathrm{A})^{+}$RNA purification used $500 \mu \mathrm{g}$ of total RNAs and the PolyA Tract mRNA Isolation System IV (Promega).

\section{Quantitation}

Quantitation used a Typhoon 9200 and the ImageQuant TL v5.0 (GE Healthcare).

\section{SUPPLEMENTAL MATERIAL}

Supplemental material can be found at http://www.rnajournal.org.

\section{ACKNOWLEDGMENTS}

We thank Ann Beyer (University of Virginia) and David Tollervey (University of Edinburgh) for stimulating discussions and useful suggestions. We are indebted to Ann Beyer and Yvonne Osheim (University of Virginia) for providing the chromatin spread (Fig. 8) and Olivier Gadal (CNRS, Toulouse), Nick Proudfoot (University of Oxford), Pierre Thurriaux (CEA, Saclay), Bertrand Séraphin (CNRS, Gif-s-Yvette), and Jean Vandenhaute (University of Namur) for kindly providing reagents. Research in the laboratory of D.L.J.L. is funded by the FNRS-FRIA, Communauté
Française (ARC convention No. 06/11-345) and Région Wallonne (CIBLES, convention RW/ULB No. 716760), and Fonds Brachet \& van Buuren.

Received October 4, 2008; accepted November 25, 2008.

\section{REFERENCES}

Abruzzi, K.C., Lacadie, S., and Rosbash, M. 2004. Biochemical analysis of TREX complex recruitment to intronless and intron-containing yeast genes. EMBO J. 23: 2620-2631.

Allmang, C., Mitchell, P., Petfalski, E., and Tollervey, D. 2000. Degradation of ribosomal RNA precursors by the exosome. Nucleic Acids Res. 28: 1684-1691.

Andersen, K.R., Jensen, T.H., and Brodersen, D.E. 2008. Take the "A" tail-Quality control of ribosomal and transfer RNA. Biochim. Biophys. Acta 1779: 532-537.

Champion, E.A., Lane, B.H., Jackrel, M.E., Regan, L., and Baserga, S.J. 2008. A direct interaction between the Utp6 halfa-tetratricopeptide repeat domain and a specific peptide in Utp21 is essential for efficient pre-rRNA processing. Mol. Cell. Biol. 27: 6547-6556.

Colau, G., Thiry, M., Leduc, V., Bordonné, R., and Lafontaine, D.L.J. 2004. The small nucle(ol)ar RNA cap trimethyltransferase is required for ribosome synthesis and intact nucleolar morphology. Mol. Cell. Biol. 24: 7976-7986.

Dez, C., Houseley, J., and Tollervey, D. 2006. Surveillance of nuclearrestricted pre-ribosomes within a subnucleolar region of Saccharomyces cerevisiae. EMBO J. 25: 1534-1546.

Dez, C., Dlakic, M., and Tollervey, D. 2007. Roles of the HEAT repeat proteins Utp10 and Utp20 in 40S ribosome maturation. RNA 13: $1516-1527$.

Dosil, M. and Bustelo, X.R. 2004. Functional characterization of Pwp2, A WD family protein essential for the assembly of the $90 \mathrm{~S}$ pre-ribosomal particle. J. Biol. Chem. 279: 37385-37397.

Dragon, F., Gallagher, J.E., Compagnone-Post, P.A., Mitchell, B.M., Porwancher, K.A., Wehner, K.A., Wormsley, S., Settlage, R.E., Shabanowitz, J., Osheim, Y., et al. 2002. A large nucleolar U3 ribonucleoprotein required for $18 \mathrm{~S}$ ribosomal RNA biogenesis. Nature 417: 967-970.

Dziembowski, A., Lorentzen, E., Conti, E., and Seraphin, B. 2007. A single subunit, Dis3, is essentially responsible for yeast exosome core activity. Nat. Struct. Mol. Biol. 14: 15-22.

Gallagher, J.E., Dunbar, D.A., Granneman, S., Mitchell, B.M., Osheim, Y., Beyer, A.L., and Baserga, S.J. 2004. RNA polymerase I transcription and pre-rRNA processing are linked by specific SSU processome components. Genes \& Dev. 18: 2506-2517.

Grandi, P., Rybin, V., Bassler, J., Petfalski, E., Strauss, D., Marzioch, M., Schafer, T., Kuster, B., Tschochner, H., Tollervey, D., et al. 2002. 90 S pre-ribosomes include the $35 \mathrm{~S}$ pre-rRNA, the U3 snoRNP, and $40 \mathrm{~S}$ subunit processing factors but predominantly lack 60S synthesis factors. Mol. Cell 10: 105-115.

Harnpicharnchai, P., Jakovljevic, J., Horsey, E., Miles, T., Roman, J., Rout, M., Meagher, D., Imai, B., Guo, Y., Brame, C.J., et al. 2001. Composition and functional characterization of yeast $66 \mathrm{~S}$ ribosome assembly intermediates. Mol. Cell 8: 505-515.

Henras, A.K., Soudet, J., Gerus, M., Lebaron, S., CaizerguesFerrer, M., Mougin, A., and Henry, Y. 2008. The post-transcriptional steps of eukaryotic ribosome biogenesis. Cell. Mol. Life Sci. 65: 2334-2359.

Houseley, J. and Tollervey, D. 2006. Yeast Trf5p is a nuclear poly(A) polymerase. EMBO Rep. 7: 205-211.

Houseley, J., LaCava, J., and Tollervey, D. 2006. RNA-quality control by the exosome. Natl. Rev. 7: 529-539.

Houseley, J., Kotovic, K., El Hage, A., and Tollervey, D. 2007. Trf4 targets ncRNAs from telomeric and rDNA spacer regions and functions in rDNA copy number control. EMBO J. 26: 4996-5006. 
Jorgensen, P., Tyers, A., and Warner, J.R. 2004. Forging the factory: Ribosome synthesis and growth control in budding yeast. In Cell growth: Control of cell size, (eds. M.N. Hall et al.), pp. 329-370. Cold Spring Harbor Laboratory Press, Cold Spring Harbor, NY.

Krogan, N.J., Peng, W.T., Cagney, G., Robinson, M.D., Haw, R., Zhong, G., Guo, X., Zhang, X., Canadien, V., Richards, D.P., et al. 2004. High-definition macromolecular composition of yeast RNAprocessing complexes. Mol. Cell 13: 225-239.

Kuai, L., Fang, F., Butler, J.S., and Sherman, F. 2004. Polyadenylation of rRNA in Saccharomyces cerevisiae. Proc. Natl. Acad. Sci. 101: 8581-8586.

Kwapisz, M., Wery, M., Despres, D., Ghavi-Helm, Y., Soutourina, J., Thuriaux, P., and Lacroute, F. 2008. Mutations of RNA polymerase II activate key genes of the nucleoside triphosphate biosynthetic pathways. EMBO J. 27: 2411-2421.

LaCava, J., Houseley, J., Saveanu, C., Petfalski, E., Thompson, E., Jacquier, A., and Tollervey, D. 2005. RNA degradation by the exosome is promoted by a nuclear polyadenylation complex. Cell 121: 713-724.

Lebreton, A. and Seraphin, B. 2008. Exosome-mediated quality control: Substrate recruitment and molecular activity. Biochim. Biophys. Acta 1779: 558-565.

Lebreton, A., Tomecki, R., Dziembowski, A., and Seraphin, B. 2008. Endonucleolytic RNA cleavage by a eukaryotic exosome. Nature 456: 993-996. doi: 10.1038/nature07575.

Mougey, E.B., O’Reilly, M., Osheim, Y., Miller, O. L., T Beyer, A., and Sollner-Webb, B. 1993. The terminal balls characteristic of eukaryotic rRNA transcription units in chromatin spreads are rRNA processing complexes. Genes \& Dev. 7: 1609-1619.

Osheim, Y.N., French, S.L., Keck, K.M., Champion, E.A., Spasov, K., Dragon, F., Baserga, S.J., and Beyer, A.L. 2004. Pre-18S ribosomal RNA is structurally compacted into the SSU processome prior to being cleaved from nascent transcripts in Saccharomyces cerevisiae. Mol. Cell 16: 943-954.

Perez-Fernandez, J., Roman, A., De Las Rivas, J., Bustelo, X.R., and Dosil, M. 2007. The 90 S preribosome is a multimodular structure that is assembled through a hierarchical mechanism. Mol. Cell. Biol. 27: 5414-5429.

Peyroche, G., Milkereit, P., Bischler, N., Tschochner, H., Schultz, P., Sentenac, A., Carles, C., and Riva, M. 2000. The recruitment of RNA polymerase I on rDNA is mediated by the interaction of the A43 subunit with Rrn3. EMBO J. 19: 5473-5482.

Prescott, E.M., Osheim, Y.N., Jones, H.S., Alen, C.M., Roan, J.G., Reeder, R.H., Beyer, A.L., and Proudfoot, N.J. 2004. Transcriptional termination by RNA polymerase I requires the small subunit Rpa12p. Proc. Natl. Acad. Sci. 101: 6068-6073.

Prieto, J.L. and McStay, B. 2007. Recruitment of factors linking transcription and processing of pre-rRNA to NOR chromatin is UBF-dependent and occurs independent of transcription in human cells. Genes \& Dev. 21: 2041-2054.

Rudra, D., Mallick, J., Zhao, Y., and Warner, J.R. 2007. Potential interface between ribosomal protein production and pre-rRNA processing. Mol. Cell. Biol. 27: 4815-4824.

Schneider, C., Anderson, J.T., and Tollervey, D. 2007. The exosome subunit Rrp44 plays a direct role in RNA substrate recognition. Mol. Cell 27: 324-331.

Shimizu, K., Kawasaki, Y., Hiraga, S., Tawaramoto, M., Nakashima, N., and Sugino, A. 2002. The fifth essential DNA polymerase $\phi$ in Saccharomyces cerevisiae is localized to the nucleolus and plays an important role in synthesis of rRNA. Proc. Natl. Acad. Sci. 99: 9133-9138.

Vanacova, S., Wolf, J., Martin, G., Blank, D., Dettwiler, S., Friedlein, A., Langen, H., Keith, G., and Keller, W. 2005. A new yeast poly(A) polymerase complex involved in RNA quality control. PLoS Biol. 3: e189. doi: 10.1371/journal.pbio.0030189.

Wyers, F., Rougemaille, M., Badis, G., Rousselle, J.C., Dufour, M.E., Boulay, J., Regnault, B., Devaux, F., Namane, A., Seraphin, B., et al. 2005. Cryptic pol II transcripts are degraded by a nuclear quality control pathway involving a new poly(A) polymerase. Cell 121: 725-737. 

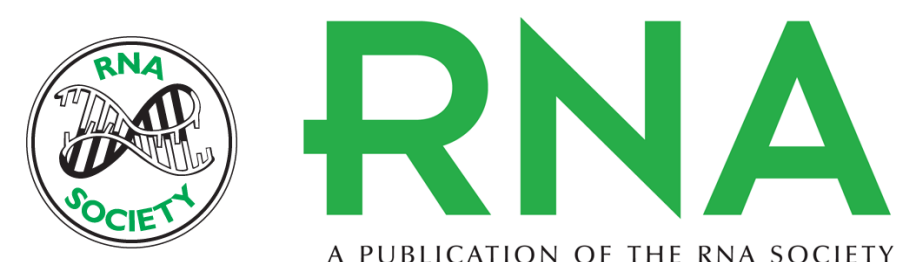

A PUBLICATION OF THE RNA SOCIETY

\section{The nuclear poly(A) polymerase and Exosome cofactor Trf5 is recruited cotranscriptionally to nucleolar surveillance}

Maxime Wery, Sabine Ruidant, Stéphanie Schillewaert, et al.

RNA 2009 15: 406-419 originally published online January 13, 2009

Access the most recent version at doi:10.1261/rna.1402709

\section{Supplemental http://rnajournal.cshlp.org/content/suppl/2009/01/13/rna.1402709.DC1}

Material

References This article cites 34 articles, 14 of which can be accessed free at: http://rnajournal.cshlp.org/content/15/3/406.full.html\#ref-list-1

Open Access Freely available online through the RNA Open Access option.

License Freely available online through the open access option.

Email Alerting Receive free email alerts when new articles cite this article - sign up in the box at the Service top right corner of the article or click here. 